\title{
Proiezioni naturali e derivazione trasversa in una varietà riemanniana a metrica iperbolica normale.
}

\author{
Memoria di Carlo Cattaneo (a Pisa)
}

A Giovanni Sansone nel swo $70^{\circ}$ compleanno.

Sunto. - Su una varietà $V_{n+1}$ a metrica iperbalica normale $(++\ldots+-)$, munita di una congruenza «temporale $C_{0}$ di riferimento, si definisce un'operazione di decomposizione saturale per un generico vettore o tensore. E'operazione viene successivamente applicata a definire una derivazione covariante trasversa (rispetto $a C_{0}$ ) operante su campi tensoriali "spaziali", con carattere invariantivo di fronte a ogni cambiamento di coordinate interno a $C_{0}$. Decomposizione naturale e derivazione trasversa vengono sistematicamente applicate all'esame differenziale di una congruenza temporale.

Scopo di questo lavorò è di illustrare in dettaglio proprietà e significato geometrico di una operazione differenziale - derivazione trasversa, ordinaria o covariante - recentemente introdotta in relatività generale $([2],[3],[4],[5])$, mostrando altresì come essa consenta, assieme all'operazione geometrica su cui essa si basa, di collegare organicamente nozioni disparate inerenti alle congruenze del genere tempo (KILLing [10], BoRN [1], Herglotz [9], Eisenhart [6], Levi-Civima [11], Gödel [8], Salzmann [16], Taub [16], Synge [17][18], Lichnerowtoz [13]).

Nelle pagine che seguono ci riferiremo a una varieta riemanniana $V_{n+1}$ a metrica iperbolica normale, con un qualsiasi numero di dimensioni. Tale generalitá, adottata anche in vista di possibili applicazioni a teorie relativistiche penta-dimensionali, non impedisce l'uso della espressiva terminologia spazio-temporale propria della relatività generale, che adopereremo sistematicamente.

I. - Decompostzione naturalie di vetwori e thensori. Protezioni-z E PROIEZIONI- $\theta$.

1. Congruenze «temporali». Coordinate adattate. Cambiamenti interni di coordinate. Sia $V_{n+1}$ una qualsiasi varietà riemanniana a metrica iperbolica normale, $x$ il generico suo punto, $T_{\infty}$ lo spazio vettoriale tangente in $x, x^{t}$ $(i=1,2, \ldots, n, 0)$ un generico sistema di coordinate locali. Sia inoltre

$$
d s^{2}=g_{i k} d x^{i} d x^{k}
$$

la forma quadratica fondamentale che supponiamo di segnatura $++\ldots++$. 
Conformemente all' uso consueto in relatività generale, si dirà del genere tempo ogni vettore $V$ di $T_{x}$ che abbia norma negativa $\left(g_{i k} V^{i} V^{k}<0\right)$, mentre ogni vettore $V$ di norma positiva $\left(g_{i k} V^{i} V^{k}>0\right)$ verrà detto del genere spazio. Le stesse locuzioni - genere tempo e genere spazio - verranno applicate anche alle direzioni dei corrispondenti vettori, mentre ai vettori di norma nulla $\left(g_{i k} V^{i} V^{k}=0\right)$ e alle rispettive direzioni verrà riservato il nome di vettori e direzioni di lunghezza nulla, ovvero quello, pure desunto dalla relatività generale, di vettori e direzioni del genere luce.

In ogni punto $x$ si dirà cono di luce, o cono elementare, l'insieme dei vettori $d P \equiv\left(d x^{i}\right)$ di $T_{x}$ aventi lunghezza nulla

$$
g_{i k} d x^{i} d x^{k}=0 .
$$

Similmente una linea di $V_{n+1}$ si dirà del genere tempo se $\dot{e}$ del genere tempo la direzione tangente in ogni suo punto. Una linea, o una qualsiasi sottovarietà di $V_{n+1}$, si dirà del genere spazio se ogni vettore ad essa tangente d̀ del genere spazio. Si dirà infine del genere luce una linea 0 sottovarietà di $V_{n+1}$ in cui ogni vettore tangente abbia lunghezza nulla, e sia pertanto tangente in ogni suo punto $x$ al corrispondente cono elementare.

i immediato riconoscere che condizione necessaria e sufficiente perchè una ipersuperficie $W_{n}$ di $V_{n+1}$ sia del genere spazio è che la sua normale sia ovunque del genere tempo.

Un sistema di coordinate locali $x^{i}$ si dirà adatlato al carattere iperbolico normale della metrica di $V_{n+1}$ se le linee coordinate $x^{0}=$ var. sono del genere tempo e le varieta coordinate $x^{0}=$ cost. sono del genere spazio. In un tal sistema di coordinate la variabile $x^{0}$ (che noi preferiamo considerare l'ultima anzichè la prima) sarà detta coordinata temporale, mentre le altre, che indicheremo globalmente con $x^{\alpha}$, saranno dette coordinate spaziali (conveniamo una volta per tutte che gli indici greci varino soltanto da 1 ad $n$, riservando agli indici latini la possibilita di assumere anche il valore 0). Un sistema di coordinate adattato al carattere dellà metrica si dirà anche, con locuzione desunta dalla relatività generale, un sistema di coordinate fisicamente ammis. sibili." L'adozione di tali coordinate, che noi da ora in poi sottintenderemo, comporta notoriamente la condizione $g_{00}<0$ e il carattere definito positivo della forma quadratica ternaria $g_{\alpha \mathrm{p}} d x^{\alpha} d x^{\beta}$, con tutte le condizioni algebriche che essa implica:

$$
g_{00}<0, \quad g_{\alpha \beta} d x^{\alpha} d x^{\beta}>0
$$

$\left(d x^{\alpha}\right.$ non simultaneamente nulli).

Scelto ad arbitrio un sistema di coordinate $x^{i}$ adattate al carattere iperbolico normale della metrica, la congruenza $C_{0}$ delle linee temporali $x^{0}=$ var. verrà detta congruenza principale di riferimento, nel dato sistema di coordinate. In relativita generale le linee della congruenza principale sono interpretabili come le linee orarie di altrettante particelle ideali di riferimento, costituenti 
il riferimento fisico associato al sistema prescelto di coordinate (cfr. MoLLER [14], p. 233).

Viceversa, scelta a piacere una congruenza $C_{0}$ di linee del genere tempo, è sempre possibile, e in infinite maniere, associare ad essa un sistema di coordinate adattate che ammetta $C_{0}$ come congruenza principale.

Un cambiamento di coordinate che lasci ferme le linee coordinate temporali, cioè la congruenza principale di riferimento, verrà detto un cambiamento di coordinase interno ulla congruenza medesima (in relatività generale un tal cambiamento si dice, in modo più espressivo, interno al riferimento fisico). Il più generale cambiamento interno è del tipo

$$
x^{\alpha^{\prime}}=x^{x^{\prime}}\left(x^{1}, x^{2}, \ldots, x^{n}\right), \quad x^{0^{\prime}}=x^{0^{\prime}}\left(x^{1}, x^{2}, \ldots, x^{n}, x^{0}\right)
$$

$\left(\partial x^{0^{t}} / \partial x^{0}>0\right)$ e può evidentemente decomporsi nel prodotto di una trasformazione delle sole coordinate spaziali

$$
x^{x^{\prime}}=x^{x^{\prime}}\left(x^{1}, x^{2}, \ldots, x^{n}\right), \quad x^{0^{\prime}}=x^{0},
$$

per una trasformazione che muti la sola coordinata temporale

$$
x^{x^{\prime}}=x^{a}, \quad x^{0^{\prime}}=x^{0^{\prime}}\left(x^{1}, x^{2}, \ldots, x^{n}, x^{0}\right) .
$$

È ben prevedibile che lo studio di una qualsiasi congruenza temporale $C_{0}$, cosi come lo studio della varietà $V_{n+1}$ in relazione alla congruenza medesima, saranno agevolati dall' uso di coordinate che ammettano la $C_{0}$ come con. gruenza principale.

Fissato in $V_{n+1}$ un sistema di coordinate locali $x^{i}$, o anche soltanto la sua congruenza principale di riferimento $C_{0}$, resta definito univocamente in $V_{n+1}$ il campo $\gamma(x)$ dei vettori unitari tangenti alle linee $x^{0}$ e orientati nel futuro (cioè nel senso delle $x^{0}$ crescenti), campo che a sua volta individua completamente la $C_{0}$. In coordinate adattate le componenti di $\gamma$, contravarianti e covarianti, valgono rispettivamente

$$
\left\{\begin{array}{l}
\gamma^{\alpha}=0, \quad \gamma^{0}=1 / \sqrt{-g_{00}} \\
\gamma_{i}=g_{i 0} / \sqrt{-g_{00}} .
\end{array}\right.
$$

Al vettore unitario $\gamma$, che nelle considerazioni che seguono avrà una parte essenziale, daremo il nome di vettore temporale di riferimento nel punto $x$.

In $T_{x}$, spazio vettoriale tangente alla $V_{n+1}$ nel punto $x$, indichiamo con $\theta_{\infty}$ il sottospazio unidimensionale dei vettori collineari a $\gamma$, e con $\Sigma_{x}$ il sottospazio tridimensionale, supplementare del precedente, ortogonale a $\gamma$. Per analogia col caso della relativită generale, in cui $T_{\infty}$ ha la struttura di uno spazio di MrNkowskJ, ai due sottospazi $\Theta_{\infty}$ e $\Sigma_{x}$, tra loro ortogonali e supplementari, daremo i nomi rispettivi di asse temporale e di piattaforma spaziale, tra loro associati. Spesso verrà sottinteso, per entrambi, il suffisso $x$.

In conformità con le definizioni ora date, e senza confusione con le 
locuzioni più generiohe introdotte in precedenza, diremo puramente temporali i vettori di $\Theta_{\infty}$ e puramente spaziali i vettori di $\Sigma_{x}$.

In coordinate adattate $i$ vettori puramente temporali sono caratterizzati dall'avere nulle le prime $n$ componenti contravariauti (confr. per esempio la (1.7)); i vettori puramente spaziali sono invece caratterizzati dall'aver nulla la $(n+1)$-ma componente covariante. In modo analogo, anche per un tensore di ordine $\geq 2$, un indice si diri puramente temporale se sono nulle tutte le componenti del tensore che abbiano quell' indice, pensato di contravarianza, diverso da 0 . Un indice si dirà invece puramente spaziale se sono nulle tutte le componenti del tensore che abbiano quell'indice, pensato di covarianza, uguale a 0 . Tali locuzioni relative a tensori d'ordine superiore al primo, verranno meglio ginstificate in seguito.

È quasi superfluo aggiangere che tutte le definizioni e locuzioni precedenti sono relative al riferimento prescelto, o meglio alla sua congruenza principale $C_{0}$.

2. Decomposizione naturale di un generico vettore. Proiezioni- $\Theta$ e proiezioni-s. Come è ben noto, un qualsiasi vettore di uno spazio vettoriale $E$ è univocamente decomponibile nella somma di vettori rispettivamente appartenenti ad assegnati sottospazi di $E$ tra loro supplementari. In particolare per ogni vettore $V$ di $T_{x}$, spazio vettoriale tangente alla $V_{n+1}$ nel punto $x$, è univocamente determinata la decomposizione

$$
\boldsymbol{V}=\boldsymbol{A}+\boldsymbol{N}
$$

con $\boldsymbol{A}$ appartenente a $\Theta_{x}$ ed $N$ a $\Sigma_{x}$. Precisamente si ha, come si può subito controllare

$$
\boldsymbol{A}=-(\boldsymbol{V} \cdot \gamma) \gamma \quad \boldsymbol{N}=\boldsymbol{V}+(\boldsymbol{V} \cdot \gamma) \gamma
$$

ovvero, in termini di componenti naturali (cioè secondo la base indotta in $T_{x}$ dal sistema di coordinate $x^{i}$; cfr. in proposito il successivo $\mathbf{n}$. 3 )

$$
A_{i}=-\gamma_{i \gamma_{k}} V^{k} \quad N_{i}=\left(g_{i k}+\gamma_{i} \gamma_{k}\right) V^{k}=\gamma_{i k} V^{k}
$$

ove si è posto

$$
\gamma_{i k}=g_{i k}+\gamma_{i} \gamma_{k} .
$$

Siffatta decomposizione di $\boldsymbol{V}$ nella somma di un vettore puramente temporale (parallelo a $\gamma$ ) e di un vettore puramente spaziale (ortogonale a $\gamma$ ) verra da ora in poi chiamata decomposizione naturale di $V$.

I due vettori componenti $\boldsymbol{A}$ ed $\boldsymbol{N}$ si diranno rispettivamente la proiesione temporale e la proiezione spaziale di $\boldsymbol{V}$. Inoltre, estendendo ad una varietà generica $V_{n+1}$ una locuzione già parzialmente in uso in relatività generale (cfr. LIohnenowicz [13] p. 7) chiameremo norma temporale e norma spaziale 
del vettore $V$, rispetto al vettore temporale di riferimento $\gamma$, la norma spa. zio-temporale dei vettori $\boldsymbol{A}$ ed $\boldsymbol{N}$, rispettivamente:

$$
\begin{cases}\|\boldsymbol{V}\|_{\Theta}=g_{i k} A^{i} A^{k}=-\gamma_{i \gamma_{k}} V^{i} V^{k} & (<0) \\ \|\boldsymbol{V}\|_{\mathbf{\Sigma}}=g_{i k} N^{i} N^{k}=\gamma_{i k} V^{i} V^{k} & (>0) .\end{cases}
$$

Nelle (2.5), come nelle (2.3), appare l'intervento sistematico dei due tensori doppi simmetrici $-\gamma_{i} \gamma_{k}$ e $\gamma_{i k}$ (definito dalla (2.4)) che del vettore $\boldsymbol{V}$ mettono in evidenza, rispettivamente, il contenuto temporale e il contenuto spaziale. Palesemente ciascuno di essi compie nelle (2.3) una funzione diversa che nelle (2.5): in quest' ultima essi compiono un ufficio metrico mentre nelle (2.3) essi, operando per semplice composizione, hanno per effetto di proiettare il vettore $V$ rispettivamente su $\theta_{x}$ e su $\Sigma_{x}$. In relazione a tale duplice ufficio noi chiameremo $-\gamma_{i} \gamma_{k}$ tensore metrico temporale o proiettore temporale nel punto $x$, riservando invece a $\gamma_{i k}$ le denominazioni di tensore metrico spaziale o proiettore spaziale. Vedremo tra poco come tali uffici dei due tensori metrici $-\gamma_{i \gamma_{k}}$ e $\gamma_{i k}$ si estendano dal caso di un semplice vettore $\boldsymbol{V}$ al caso di un tensore di qualsiasi ordine, restando cosi ulteriormente giustificate le loro denominazioni. Esamineremo anche, tra breve, la loro struttura e le loro principali proprietà.

3. Base naturale in $T_{x}$ e basi naturalmente indotte in $\Sigma_{x}$ e in $\Theta_{x}$. Appli. cheremo immediatamente le operazioni di proiezione spaziale e temporale per definire una base naturale in $\Sigma_{x}$ e in $\Theta_{x}$.

Come è noto, il sistema coordinato $x^{i}$ induce nello spazio vettoriale tangente $T_{x}$ una base naturale, i cui vettori ( $n$ del genere spazio e uno del genere tempo, rispettivamente tangenti alle singole linee coordinate) indicheremo con $\partial_{i} P$. Le componenti contravarianti e covarianti di $\partial_{i} P$ sono rispettivamente

$$
\left(\partial_{i} P\right)^{h}=\delta_{i}{ }^{h}, \quad\left(\partial_{i} P\right)_{h}=g_{h k} \delta_{i}{ }^{k}=g_{h i}
$$

$\delta_{i}{ }^{h}$ essendo l'usuale simbolo di KRoNECKER. La precedente notazione si giu. stifica osservando che se, come è nell' uso, si indica con $d P \equiv\left(d x^{i}\right)$ il generico vettore dello spazio tangente $T_{x}$, risulta $d P=\partial_{i} P d x^{i}$, proprio come se $a_{i}$ e $d$ indicassero rispettivamente derivazioni parziali e differenziazione totale.

In conformita alla precedente definizione di base naturale, in $T_{x}$, si diranno componenti naturali, contravarianti o covarianti, di un vettore o tensore, le sue componenti, dell'una o dell'altra specie, secondo la base naturale.

Cio premesso, appare spontaneo chiamare base naturale della piattaforma spaziale $\Sigma_{\infty}$ l'insieme delle $n$ proiezioni ortogonali su $\Sigma_{x}$ - che indiche. remo $\tilde{\partial}_{\alpha} P$ - dei primi $n$ vettori $\partial_{\alpha} P$ della base naturale di $T_{x}$. Stante la $(3.1)$ 
e per quanto si è detto al n. 2, è immediato ricavare le $n+1$ componenti di $\tilde{\partial}_{i} P$ nella base naturale di $T_{x}$ :

$$
\left\{\begin{array}{l}
\left(\tilde{\partial}_{\alpha} P\right)_{i}=\gamma_{i k} \delta_{\alpha}^{k}=\gamma_{i \alpha} \\
\left(\tilde{\partial}_{\alpha} P\right)^{i}=g^{i h_{\gamma_{\alpha}}}=\delta_{\alpha}^{i}+\gamma^{i} \gamma_{\alpha} .
\end{array}\right.
$$

In forma vettoriale gli $n$ vettori della base naturale in $\Sigma_{x}$ si esprimono per mezzo degli $n+1$ vettori della base naturale in $T_{\infty}$ al modo seguente:

$$
\tilde{\partial}_{\alpha} P=\partial_{\alpha} P+\gamma_{\alpha} \gamma^{0} \partial_{0} P \text {. }
$$

Anticipando un poco, diremo che l'espressione che compare a secondo membro coincide formalmente con quella di un'operazione differenziale che verrà introdotta più avanti (n. 6).

Quanto al sottospazio temporale $\Theta_{x}$, ad una sola dimensione, diremo sua base naturale il vettore $\partial_{0} P$, ciò l'unico vettore del genere tempo della base naturale di $T_{x}$. Questo, a sua volta, è legato al vettore unitario $\gamma$ precedentemente introdotto (vettore principale di riferimento) della semplice relazione

$$
\gamma=\gamma^{0} \partial_{0} P \text {. }
$$

Ciò premesso, sia $V$ un generico vettore puramente spaziale, $V^{i}$ e $V_{i} l_{e}$ sue componenti naturali in $T_{x}, V^{\tilde{\alpha}}, V_{\tilde{\alpha}}$ le sue componenti naturali in $\Sigma_{x}$; il suo carattere puramente spaziale implica:

$$
\boldsymbol{V} \cdot \gamma \equiv V_{i} \gamma^{i} \equiv V^{i} \gamma_{i}=0
$$

Dal semplice significato di componenti covarianti e dalla (3.3) segue:

$$
V_{\tilde{\alpha}}=V \cdot \tilde{\partial}_{\alpha} P=V \cdot \partial_{\alpha} P+\gamma_{\alpha} \gamma^{0} V \cdot \partial_{0} P=V_{\alpha} .
$$

Analogamente dalla duplice identità $V \equiv V^{\alpha} \tilde{\partial}_{\alpha} P, V \equiv V^{i} \partial_{i} P$ e dalla (3.3), tenuto anche conto della (3.6), discende:

$$
V^{\tilde{\alpha}}=V^{\alpha} \text {. }
$$

La (3.6) e la (3.7) si riassumono nella seguente osservazione di utilità pratica: le componenti, covarianti o controvarianti, di un vettore puramente spaziale secondo la base naturale di $\Sigma_{\infty}$ si identificano con le sue prime $n$ componenti, rispettivamente covarianti o controvarianti, secondo la base naturale di $T_{x}$.

E pure immediato controllare che le componenti naturali covarianti del tensore metrico di $\Sigma_{x}$, date dagli $n^{2}$ prodotti scalari $\tilde{\partial}_{\alpha} P \cdot \tilde{\partial}_{\beta} P$, si jdentificano con le $\gamma_{x \beta}$.

Quanto al tensore metrico in $\theta_{\infty}$, che peraltro non ha un grande interesse, esso ha un' unica componente covariante data dal prodotto scalare $\partial_{0} P \cdot \partial_{0} P$ e si identifica pertanto con la componente $g_{00}$ del tensore fondamentale di $V_{n+1}$. La sua unica componente contravariante vale $1 / g_{00}$. 
C. Cattaneo: Proiezioni naturali e derivazione trasversa, ecc.

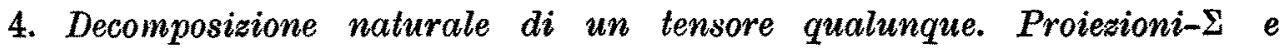
proiezioni- $\Theta$. Carattere puramente spaziale o temporale di un indice.

La decomposizione simbolica di $T_{x}$

$$
T_{x}=\Sigma_{x}+\Theta_{x}
$$

induce in $T_{x} \otimes T_{x}$ una decomposizione nella somma di quattro sottospazi mutuamente ortogonali e complessivamente supplementari

$$
T_{x} \otimes T_{x}=\Sigma_{x} \otimes \Sigma_{x}+\Sigma_{x} \otimes \Theta_{x}+\Theta_{x} \otimes \Sigma_{x}+\theta_{x} \otimes \Theta_{x} .
$$

Ne segue che un generico tensore doppio $t_{i j}$ è univocamente decomponibile nella somma di quattro tensori a due a due ortogonali $\theta$ appartenenti rispettivamente ai quattro sottospazi ora nominati. Tale decomposizione verrà chiamata la decomposizione naturale del tensore $t_{i j}, \theta \mathrm{i}$ singoli tensori componenti saranno chiamati proiezioni naturali del tensore $t_{i j}$ sui rispettivi sottospazi. Tali proiezioni verranno simbolicamente indicate cosi : $\mathfrak{S}_{\Sigma \Sigma}\left(t_{i j}\right), \mathfrak{S}_{\Sigma \Theta}\left(t_{i j}\right)$, $\mathfrak{S}_{\Theta \Sigma}\left(t_{i j}\right), \mathfrak{S}_{\Theta \Theta}\left(t_{i j}\right), \mathfrak{S}$ indicando genericamente un operatore di proiezione.

Il procedimento formale onde ottenere da un dato tensore le sue varie proiezioni naturali è quanto mai semplice: si opera su ciascun indice mediante il conveniente proiettore, $\gamma_{i k} \circ-\gamma_{i} \gamma_{k}$, a seconda che l'indice medesimo debba trasformarsi in un indice spaziale $o$ in un indice temporale:

$$
\begin{array}{ll}
\mathfrak{S}_{\Sigma \Sigma}\left(t_{i j}\right)=\gamma_{i r} \gamma_{j s} t^{r s}, & \mathscr{S}_{\Sigma \oplus(}\left(t_{i j}\right)=-\gamma_{i r} \gamma_{j} \gamma_{s} t^{r s}, \\
\mathfrak{S}_{\Theta \Sigma}\left(t_{i j}\right)=-\gamma_{i} \gamma_{r} \gamma_{j s} t^{r s}, & \mathscr{B}_{\Theta \Theta(}\left(t_{i j}\right)=\gamma_{i} \gamma_{j} \gamma_{r} \gamma_{s} t^{r s} .
\end{array}
$$

Come esempio, interessante se pur banale, si possono calcolare le varie pro. iezioni naturali del tensore fondamentale $g_{i j}$ :

$$
\begin{array}{ll}
\mathfrak{B}_{\Sigma \Sigma}\left(g_{i j}\right)=\gamma_{i j}, & \mathfrak{S}_{\Sigma \Theta}\left(g_{i j}\right)=0 \\
\mathfrak{B}_{\Theta \Sigma}\left(g_{i j}\right)=0, & \mathfrak{S}_{\Theta \Theta}\left(g_{i j}\right)=-\gamma_{i} \gamma_{j} .
\end{array}
$$

Resta confermato così che le proiezioni spaziale e temporale di $g_{i j}$ coincidono col tensore metrico di $\Sigma$ e di $\theta$, rispettivamente, e che il criterio di indurre una metrica in un sottospazio definendo come base indotta la proiezione dei primi $n$ vettori della base di $T_{\infty}$ e successivamente calcolando i vari prodotti scalari $\tilde{\partial}_{x} P \cdot \tilde{\partial_{\beta}} P$, coincide col criterio della proiezione diretta di $g_{i j}$.

Un indice, indifferentemente di covarianza o di contravarianza, di un generico tensore si dirà avere carattere puramente spaziale se il tensore medesimo non viene alterato operando su quell'indice mediante il proiettore spaziale $\gamma_{i k}$. Un indice si dirì invece avere carattere puramente temporale se il tensore non viene modificato operando su quell'indice mediante il pro. iettore temporale $-\gamma_{i} \gamma_{k}$. In accordo con quanto gia si disse al $\mathrm{n}$. 1 , sono nulle tutte le componenti di un tensore in cui un indice puramente spaziale, 
in posizione di covarianza, assuma il valore 0. Dal pari sono nulle tatte le componenti di un tensore allorchè un indice puramente temporale, in posi. zione di contravarianza, assume un qualsiasi valore diverso da 0 .

Un tensore doppio si dirà totalmente spaziale se esso appartiene a $\Sigma \otimes \Sigma$, cioè se entrambi i suoi indici sono puramente spaziali; si dirà all'opposto totalmente temporale se esso appartiene a $\Theta \otimes \Theta$, vale a dire se entrambi i suoi indici sono puramente temporali.

Quanto precede si estende in modo ovvio a tensori di ordine qualunque; per i quali si parlera di proiezioni del tipo $\mathfrak{S}_{\mathrm{E} \Theta_{\ldots} . . \mathrm{Z}}\left(t_{i j \ldots . . .}\right)$ con tanti indici, nguali a $\Sigma$ o a $\theta$, quanti sono gli indici del tensore.

\section{Qualche proprietà algebrica dell'operazione di proiezione.}

a) Allorchè la proiezione- $\Sigma$ opera su di un indice puramente temporale essa dà per risultato ì tensore nullo.

b) Allorchè la proiezione- $\Theta$ opera su di un indice puramente spaziale essa da per risultato il tensore nullo.

c) Allorchè un'operazione di proiezione completa, ciò̀ operante su tutti gli indici, opera sul prodotto di due o più tensori (senza contrazioni) essa si ripartisce tra i fattori, rispettando l'ordine di corrispondenza tra gli indici: $\mathfrak{B}_{\Sigma \otimes \mathbb{\Sigma}}\left(A_{i} B_{j r}\right)=\mathfrak{B}_{\mathbb{\Sigma}}\left(A_{i}\right) \mathfrak{B}_{\Theta \mathbb{E}}\left(B_{j r}\right)$.

d) La decomposizione naturale, applicata ad un generico tensore $A_{i j}$ può seriversi :

$$
A_{i j}=\tilde{A}_{i j}+\tilde{A}_{i \gamma_{j}}+\gamma_{i} \tilde{A}_{j}^{\prime}+A \gamma_{i \gamma_{j}}
$$

ove si è posto

$$
\begin{array}{ll}
\tilde{A}_{i j}=\gamma_{i r} \gamma_{i s} A^{r s}, & \tilde{A}_{i}=-\gamma_{i r} \gamma_{s} A^{r s} \\
\tilde{A}_{j}^{\prime}=-\gamma_{r}{ }^{\gamma} j_{s} A^{r s}, & A=\gamma_{r} \gamma_{s} A^{4 s} .
\end{array}
$$

Un generico tensore doppio $A_{i j}$ risulta pertanto individuato da un tensore totalmente spaziale $\tilde{A}_{i j}$; da due vettori $\tilde{A}_{i}$ e $\tilde{A}_{i}^{\prime}$ puramente spaziali, e da uno scalare $A$, cui corrispondono complessivamente appunto 16 quantita numeriche indipendenti.

Se il tensore $A_{i j}$ è simmetrico, tale è anche il tensore $\tilde{A}_{i j}$, mentre $\mathrm{i}$ due vettori $\tilde{A}_{i}$ e $\tilde{A}_{i}^{\prime}$ coincidono. Per un tensore simmetrico $S_{i j}=S_{j i}$ può dunque scriversi

$$
S_{i j}=\tilde{S}_{i j}+\tilde{S}_{i \gamma_{j}}+\gamma_{i} \tilde{S}_{j}+S_{\gamma_{i} \gamma_{j}} \quad\left(\tilde{S}_{i j}=\tilde{S}_{j i}\right)
$$

Se poi il tensore che viene decomposto è antisimmetrico, $\Omega_{i j}=-\Omega_{i_{j}}$, anche il tensore $\tilde{\Omega}_{i j}$ risulta antisimmetrico, i dae vettori spaziali risultano 
l' uno l'opposto dell'altro, mentre infine lo scalare si annulla. La decomposizione naturale di un tensore antisimmetrico $\Omega_{i j}=-\Omega_{j i}$ si scriverà pertanto

$$
\boldsymbol{\Omega}_{i j}=\tilde{\mathbf{Q}}_{i j}+\tilde{\mathbf{\Omega}}_{i \gamma_{j}}-\gamma_{i} \tilde{\mathbf{Q}}_{j} \quad\left(\tilde{\mathbf{Q}}_{i j}=-\tilde{\mathbf{Q}}_{j i}\right) .
$$

e) La saturazione di un indice puramente spaziale con un indice pura* mente temporale (previa sistemazione dei due indici in posizioni di varianza opposta) dà per risultato lo zero, qualunque sia il numero e il carattere degli altri indici non interessati nella saturazione.

\section{II. - Derivazione trastersa.}

6. Derivazione trasversa ordinaria. Assegnato entro un dominio $D$ della varieta $V_{n+1}$ un generico campo di scalari $f\left(x^{i}\right)$ consideriamone il gradiente, grad $f$, di componenti covarianti $\hat{o}_{i} f$. Sia poi $d P \equiv\left(d x^{i}\right)$ un generico campo di vettori, soggetti all'anica condizione di essere, ovunque, perpendicolari al vettore $\gamma$, cio che implica $\gamma_{i} d x^{i}=0$, ovvero

$$
d x^{0}=-\frac{1}{\gamma_{0}} \gamma_{\alpha} d x^{\alpha} \equiv \gamma^{0} \gamma_{\alpha} d x^{x}
$$

Ciò premesso, il differenziale totale di $f$ secondo il vettore $d P$ si esprime: $d f=\partial_{i} f d x x^{i}$, ovvero, tenuto conto della condizione (6.1), nell' uno o nell' altro dei due modi seguenti :

$$
d f=\left(\partial_{\alpha} f-\frac{\gamma_{\alpha}}{\gamma_{0}} \partial_{0} f\right) d x^{\alpha} \equiv\left(\partial_{\alpha} f+\gamma^{0} \gamma_{\alpha} \partial_{0} f\right) d x^{\alpha},
$$

ove l'indice di sommazione varia ora soltanto da 1 a $n$. Nasce cosi in modo spontaneo l'operatore differenziale, che indicheremo semplicemente con $\tilde{\partial}_{i}, \cos 1$ definito :

$$
\tilde{\partial}_{i}=\partial_{i}-\frac{\gamma_{i}}{\gamma_{0}} \partial_{0} \equiv \partial_{i}+\gamma_{i} \gamma^{0} \partial_{0}
$$

operatore che avrà molta importanza nel seguito. Mediante siffatto operatore, significativo soltanto per $i \neq 0$, il differenziale totale di $f$ secondo un vettore $d P$ normale a $\gamma$, può dunque esprimersi anche con la formula

$$
d f=\tilde{\partial}_{i} f d x^{i} \equiv \tilde{\partial}_{a} f \cdot d s x^{x}
$$

che utilizza di fatto le sole componenti controvarianti spaziali di $d P$. La (6.3') mostra che le $n+1$ quantità $\tilde{\partial}_{i} f$, di cui l'ultima $(i=0)$ sempre nulla, sono le componenti covarianti di un vettore che, qualunque sia $f\left(x^{i}\right)$, riesce sempre 
perpendicolare a $\gamma\left(\tilde{\partial_{0}} f=0\right)$ : per tale motivo lo diremo il gradiente trasverso (rispetto alla direzione di $\gamma$ ) del campo scalare $f$; in simboli: grãd $f \equiv\left(\tilde{\partial}_{i} f\right)$.

Fatto notevole è che l'ora definitc gradiente trasverso non è che la proiezione dell'ordinario gradiente sopra la piattaforma spaziale $\mathbf{\Sigma}$. Si ha infatti $\tilde{\partial}_{i} f=\left(\delta_{i}{ }^{n}+\gamma_{i} \gamma^{h}\right) \partial_{h} f$ e quindi

$$
\tilde{\partial}_{i} f=\mathfrak{B}_{\Sigma}\left(\partial_{i} f\right)
$$

Tutto ciò giustifica per l'operazione $\tilde{\mathfrak{a}}_{i}$ definita dalla (6.3), il nome di derivazione parziale trasversa. La denominazione è ulteriormente giustificata dalla seguente interpretazione. Se si tengono presenti ad un tempo sia la definizione formale (6.3) sia la (3.2), si riconosce immediatamente che $\tilde{\partial}_{\alpha}$ non è che l'operazione di differenziazione totale secondo l' $\alpha$-mo vettore della base naturale indotta in $\Sigma_{x}$.

È quasi evidente che per l'operazione $\tilde{\mathfrak{c}}_{i}$ valgono la maggior parte delle proprietà formali di cui gode la derivazione parziale ordinaria, in particolare la regola di derivazione di una somma, di un prodotto, di nn quoziente ece.

L' operazione di derivazione parziale trasversa può essere applicata più volte successivamente, rispetto a valori qualunque dell'indice, dando così luogo a derivazioni trasverse di secondo ordine o di ordini superiori. Tuttavia, come si riconosce immediatamente, l'ordine delle derivazioni successive generalmente non è permutabile. Ci accontentiamo per ora di questo risultato formale negativo, riservandoci di esaminare più avanti $i$ casi speciali in cui il prodotto $\tilde{\partial}_{\beta} \tilde{\partial}_{\alpha}$ e commutativo e delle condizioni che tale commutativita implica per la congruenza prineipale $C_{0}$.

In modo immediato si può riconoscere che l'operatore $\tilde{\partial}_{\alpha}$ non è general. mente commutabile neppure con l'operatore $\partial_{0}$.

7. Derivazione longitudinale $\bar{\partial}_{0}$. Consideriamo ancora il campo di vettori grad $f$, e punto per punto del dominio $D$, proiettiamolo ortogonalmente su $\Theta_{x}$ : la componente algebrica del vettore proiezione secondo il vettore base di $\Theta_{x}\left(\partial_{0} P\right)$ chiameremo derivata longitudinale (secondo la direzione di $\gamma$ ) del campo di scalari $f$ e indicheremo con $\bar{\partial}_{0} f$ :

$$
\bar{\partial}_{0} f=\gamma^{0} \partial_{0} f
$$

Essa differisce dalla semplice derivata parziale rispetto a $x^{0}$ per il fattore $\gamma^{0}$. Naturalmente anche l'operatore $\overline{\partial_{0}}$ non è generalmente commutabile nè con $\tilde{\partial}_{\alpha}$ nè $\operatorname{con} \partial_{0}$.

Non sarà inutile aggiungere che l'operatore $\bar{\partial}_{0}$, come l'operatore $\tilde{\partial}_{\alpha}$, è invariante di fronte ad ogni cambiamento di coordinate interno alla congruenza $C_{0}$. 
8. Derivazione covariante trasversa di un campo di vettori puramente spaziali. L'operazione di derivazione trasversa, introdotta nei precedenti numeri, si può estendere in modo altrettanto spontaneo al caso che in luogo di un eanpo di scalari si parta da un generico campo di vettori puramente spaziali.

Sia $s(x)$ un generico campo di vettori puramente spaziali, quindi soddisfacenti alle condizioni (tra loro equivalenti)

$$
s^{0}=-\gamma_{\alpha} s^{\alpha} / \gamma_{0} \equiv \gamma^{0} \gamma_{\alpha^{s}} s^{x} ; \quad s_{0}=0 .
$$

Considerata di $s(x)$ la derivata covariante in $V_{n+1}, \nabla h s_{k}$, effettuiamone, in ogni punto di $V_{n+1}$, la proiezione totale su $\Sigma_{x}$. Il tensore doppio puramente spaziale che cosi si ottiene in ciascun punto di $V_{n+1}-\mathscr{S}_{\Sigma \Sigma}\left(\bar{\nabla}_{i} s_{j}\right)$ - chiame. remo derivata covariante travsersa del campo puramente spaziale $s(x)$. Applicando il procedimento di proiezione definito al precedente n. 4 e sviluppando i calcoli, che omettiamo, si ottiene:

$$
\mathfrak{S}_{\Sigma \Sigma}\left(\bar{\nabla}_{i} s_{j}\right)=\tilde{\partial}_{i} s_{j}-\frac{1}{2}\left(\tilde{\partial}_{i} \gamma_{j h}+\tilde{\partial}_{j} \gamma_{h i}-\tilde{\partial}_{h} \gamma_{i j}\right) s^{h}
$$

ove intervengono sistematicamente il tensore metrico spaziale $\gamma_{i j}$ e la deriva. zione trasversa $\tilde{\partial_{i}}$.

L'espressione che compare a secondo membro della (8.2) suggerisce di introdurre una nuova specie di simboli di ChrIsTOFFes, di prima o di seconda specie, formalmente costruiti come i simboli ordinari, con la materiale sostituzione del tensore metrico spaziale $\gamma_{i j}$ al tensore metrico spazio-temporale $g_{i j}$, e della derivazione parziale trasversa $\tilde{\partial}_{i}$ alla derivazione ordinaria $\tilde{\partial}_{i}$ :

$$
\widehat{\{i j, h}\}=\frac{1}{2}\left(\tilde{\partial}_{i} \gamma_{j h}+\tilde{\partial}_{j} \gamma_{h i}-\tilde{\partial}_{h} \gamma_{i j}\right)
$$

$$
\left\{\begin{array}{c}
\bar{h} \\
i j
\end{array}\right\}=g^{h r}\{\widehat{i j, r}\} \equiv \gamma^{h r}\{\widehat{i j, r}\} \text {. }
$$

Con tale introduzione la (8.2) acquista l'aspetto

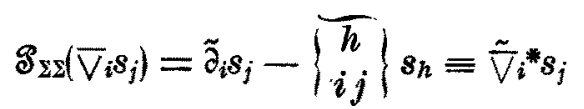

e può interpretarsi come una nuova specie di derivata covariante, che abbiamo indicato con il simbolo $\tilde{\bar{V}}_{i}^{*} s_{j}$, e che ben giustifica il nome di derivata cova. riante trasversa del campo di vettori spaziali $s_{j}$, precedentemente attribuito alla $\mathscr{S}_{\Sigma \Sigma}\left(\bar{\nabla}_{i} s_{j}\right)$. Nella notazione adottata, forse sovrabbondante, il segno * $*$ posto a ricordare l'uso delle $\gamma_{i k}$, e il segno $\sim$ l'uso della derivazione $\tilde{\partial}_{i}$.

Tanto nei simboli di Christoffel (8.3) quanto nelle componenti della derivata covariante trasversa (8.4) si riconosce immediatamente che essi si 
riducono identicamente a zero non appena uno degli indici posti in basso assume il valore 0 , ciò che conferma il carattere totalmente spaziale della derivata covariante trasversa.

Concludendo diremo che come l'operazione di derivazione ordinaria trasversa operando su di un campo di scalari dà origine ad un campo di vettori puramente spaziali (il gradiente trasverso di $f$ ), cosi l'operazione di derivazione covariante trasversa operando su un campo di vettori puramente spaziali, dà origine a un campo di tensori doppi totalmente spaziali. Formalmente la derivata covariante trasversa di un campo di vettori puramente spaziali può calcolarsi come un' ordinaria derivata covariante, usando simboli di ChristowFEL, di prima e di seconda specie, formati a partire dal tensore metrieo spaziale $\gamma_{i j}$ con l'impiego sistematico delle $\tilde{\partial}_{i}$ in luogo delle $\partial_{i}$.

9. Derivazione covariante trasversa di un tensore totalmente spaziale di ordine qualunque. L'operazione di derivazione covariante trasversa si estende agevolmente al caso di un tensore d'ordine qualnnque, purchè totalmente spaziale. Sia $s_{i j}$, ad esempio, un campo di tensori doppi totalmente spaziali $\left(s_{i 0}=0, s_{0 i}=0\right)$. Procedendo in modo perfettamente analogo a quello seguito al numero precedente, consideriamo di tal campo la derivata covariante $\bar{\nabla}_{i} s_{j m}$ e proiettiamola totalmente, punto per punto, sullo spazio $\Sigma_{x}$. Con calcoli molto semplici, benchè un poco lunghi, che qui omettiamo, si riconosce che alla cercata proiezione può darsi la forma seguente

$$
\mathfrak{S}_{\Sigma \Sigma \Sigma}\left(\bar{\nabla}_{i} s_{j m}\right)=\tilde{\partial}_{i} s_{j m}-\left\{\begin{array}{c}
\bar{h} \\
i j
\end{array}\right\} s_{h m}-\left\{\begin{array}{c}
\bar{h} \\
i m
\end{array}\right\} s_{j h} \equiv \tilde{\nabla}_{i}^{*} s_{j m} .
$$

Ancora una volta si riconosce, nel secondo membro della (9.1) una specie di dérivata covariante, da noi indicata $\tilde{\nabla}_{i}^{*} s_{j m}$, eseguita con le regole formali ordinarie, ma con l'uso sistematico della derivazione $\tilde{\partial}_{i}$ in luogo della $\partial_{i}$, e dei simboli di Christoffel (8.3) in lnogo dei simboli ordinari.

Riteniamo superfluo trattare esplicitamente il caso più generale, quanto abbiamo detto bastando ad assicurare la completa generalità dell' operazione di derivazione covariante trasversa. Essa opera su un campo di tensori totalmente spaziali e dà origine a un nuovo campo di tensori totalmente spaziali, con un indice in pí̀̀.

10. - Proprietà formali immediate della derivazione covariante trasversa. E quasi immediato riconoscere che la derivazione covariante trasversa gode di tutte le proprieta formali della derivazione covariante che sono poi lo stesse che valgono per la derivazione ordinaria: vale pertanto la derivabilità termine a termine di una somma di tensori totalmente spaziali, cosi come resta valida l'ordinaria regola di derivazione per un prodotto di più tensori 
totalmente spaziali. Si estende naturalmente alla derivazione covariante trasversa anche la proprieta di essere commutabile con l'operazione di saturazione, purchè questa operi su due indici che siano entrambi puramente spaziali.

Sussiste anche un teorema del tutto analogo al teorema di RIcor, al quale conserveremo lo stesso nome:

TeOR. DI RICOI. - La derivata covariante trasversa del tensore metrico spaziale $\gamma_{i j}$ è identicamente nulla.

La dimostrazione si svolge formalmente come la dimostrazione ordinaria.

Da questo teorema appare come il tensore metrico spaziale naturalmente indotto in $\Sigma_{x}$, trovi proprio nella derivazione covariante trasversa l'operazione differenziale che ne sottolinea il carattere di tensore spaziale fondamentale.

E quasi superfluo aggiungere che non sussiste in generale l'invertibilità di due derivazioni covarianti trasverse successive.

11. - Proiezione formale su $\mathbf{\Sigma}_{\infty}$ dei simboli di Christoffel. La tecnica della proiezione spaziale applicata nei precedenti numeri ad enti tensoriali, può formalmente applicarsi anche ad altri enti geometrici, non tensoriali, quale è ad esempio la connessione riemanniana della $V_{n+1}$, rappresentata in coordinate locali dai simboli di Christoffer di prima e di seconda specie. Cio che è veramente notevole $\dot{\theta}$ il fatto che il risultato di tale proiezione formale sono, come è facile verificare, proprio i simboli. di Christoffel modificati, definiti dalle formule (8.3) :

$$
\begin{aligned}
& \mathfrak{S}_{\Sigma \Sigma \Sigma}(\{i j, h\})=\{\widetilde{i j,} h\} \\
& \mathfrak{B}_{\mathrm{x} x}\left(\left\{\begin{array}{c}
h \\
i j
\end{array}\right\}\right)=\left\{\begin{array}{c}
\bar{h} \\
i j
\end{array}\right\} .
\end{aligned}
$$

Le (11.1), oltre a un notevole contenuto geometrico, hanno un'evidente utilità sul piano pratico, di esecuzione di calcoli.

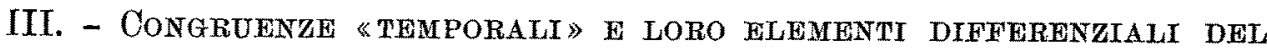
PRIMO ORDINE.

Come dicemmo all' inizio del presente lavoro (n. 1) la tecnica delle proiezioni, spaziale e temporale, e l'impiego sistematico della derivazione trasversa, ordinaria o covariante, trova utile applicazione nello studio delle congruenze di $V_{n+1}$. Fisseremo l'attenzione sulle congruenze del genere tempo, che in una varietà a metrica iperbolica normale hanno un' importanza preponderante. $L^{\prime}$ adattamento delle considerazioni che seguono al caso di una congruenza generica, non presenta però difficoltà. 
Sia $C_{0}$ una qualsiasi congruenza «temporale» di $V_{n+1}$, che assumeremo senz'altro come congruenza principale di riferimento, adottando un sistema di coordinate locali $x^{i}$ adattate alla congruenza medesima, cosi che le linee $x^{0}=$ var. si identifichino con le linee di $C_{0}$. Resta ancora disponibile, ove occorra, un cambiamento di coordinate interno alla congruenza medesima (n. 1).

La congruenza $C_{0} \dot{e}$ univocamente individuata dal campo di vettori unitari $\gamma(x)$ tangenti alle sue linee. Inizieremo pertanto l'esame locale di $C_{0}$ con l'esame dei tensori che si ricavano da $\gamma(x)$ con derivazioni del primo ordine, ciò̀ dei tre tensori seguenti: il tensore antisimmetrico $\Omega_{i j}=\partial_{i} \gamma_{j}-\partial_{j} \gamma_{i} \equiv$ $\equiv \bar{V}_{i} \gamma_{j}-\nabla_{i} \gamma_{i}$, che può definirsi il tensore vortice della congruenza; il tensore asimmetrico $\bar{\nabla}_{i} \gamma_{j}$, derivata covariante di $\gamma$, e il tensore simmetrico $K_{i j}=$ $=\nabla_{i} \gamma_{j}+\nabla_{j} Y_{i}$ che diremo il tensore di Killing di $C_{0}$.

Di tali tensori effettueremo ora la decomposizione naturale, considerando di ciascuno di essi le quattro proiezioni $\mathfrak{S}_{\Sigma \Sigma}, \mathfrak{S}_{\Sigma \Theta}, \mathfrak{S}_{\Theta \Sigma}, \mathfrak{S}_{\Theta \Theta}$. Esamineremo poi il significato geometrico di ciascuna.

12. - Le quattro proiezioni naturali del tensore vortice. - Chiamasi tensore vortice di una congruenza spazio-temporale $C_{0}$, definita da un campo di vettori unitari $\gamma(x)$, il tensore doppio antisimmetrico

$$
\Omega_{i j}=\partial_{i} \gamma_{j}-\partial_{j} \gamma_{i} \equiv \bar{\nabla}_{i} \gamma_{j}-\bar{V}_{j} \gamma_{i} .
$$

Esso è notoriamente della più grande importanza nella dinamioa relatim vistica dei fluidi (cfr. per es. LICHNerowroz [13], SrNge [17] [18] e in genere nella geometria delle congruenze. Ne esamineremo ora le quattro proiezioni naturali, delle quali vedremo più avanti il notevole significato geometrico.

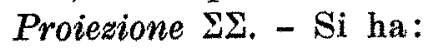

$$
\begin{aligned}
\mathfrak{S}_{\Sigma \Sigma}\left(\Omega_{i j}\right) & \equiv \tilde{Q}_{i j}=\gamma_{i r} \gamma_{j s} g^{r h} g^{s k} \boldsymbol{\Omega}_{h k} \\
& =\gamma_{0} \partial_{i} \frac{\gamma_{j}}{\gamma_{0}}-\partial_{j} \gamma_{i}+\frac{\gamma_{j}}{\gamma_{0}} \partial_{0} \gamma_{i}+\gamma_{i} \gamma_{0} \gamma^{0} \partial_{0} \frac{\gamma_{j}}{\gamma_{0}} \\
& -\gamma_{i} \gamma^{0} \partial_{j} \gamma_{0}+\gamma_{i} \gamma^{0} \frac{\gamma_{j}}{\gamma_{0}} \partial_{0} \gamma_{0} .
\end{aligned}
$$

Tenuto presente che $\gamma_{0} \gamma^{0}=-1$, l'associazione del secondo col quinto termine e del terzo col sesto dà:

$$
\mathfrak{B}_{\Sigma x}\left(\Omega_{i j}\right) \equiv \tilde{\Omega}_{i j}=\gamma_{0} \partial_{i} \frac{\gamma_{j}}{\gamma_{0}}-\gamma_{i} \partial_{0} \frac{\gamma_{j}}{\gamma_{0}}-\gamma_{0} \partial_{j} \frac{\gamma_{i}}{\gamma_{0}}+\gamma_{j} \partial_{0} \frac{\gamma_{i}}{\gamma_{0}} .
$$

Di qui infine, associando il primo col secondo termine e il terzo col quarto si trova

$$
\mathfrak{S}_{\Sigma \Sigma}\left(\boldsymbol{Q}_{i j}\right) \equiv \tilde{\mathbf{Q}}_{i j}=\gamma_{0}\left(\tilde{\partial}_{i} \frac{\gamma_{i}}{\gamma_{0}}-\tilde{a}_{j} \frac{\gamma_{i}}{\gamma_{0}}\right)
$$


Il tensore spaziale così ottenato (è immediato controllare l'annullarsi delle componenti covarianti in cui figuri almeno una volta l'indice of è un tensore antisimmetrico, come $\boldsymbol{\Omega}_{i j}$, che formalmente si ottiene come quello, sostituendo $\frac{\gamma_{i}}{\gamma_{0}}$ a $\gamma_{i}$ e l'operazione $\tilde{\partial}_{i}$ alla $\partial_{i}$, con in più la moltiplicazione per $\gamma_{0}$. Tale tensore chiameremo il tensore vortice spaziale, o rotore trasverso, della congruenza $C_{0}$ (o del campo di vettori $\left.\gamma(x)\right)$.

Proiezione-5Q. - Si ha:

$$
\mathscr{S}_{\mathbb{\Sigma} \Theta}\left(\Omega_{i j}\right)=-\gamma_{i}{ }^{r} \gamma_{j} \gamma^{s} \Omega_{r s}=\gamma^{0} \Omega_{0 i} \gamma_{j}
$$

o anche, osservando ohe

$$
\gamma^{0} \Omega_{0 i}=\gamma^{r} \Omega_{r i}=\gamma^{r}\left(\bar{\nabla}_{r} \gamma_{i}-\bar{\nabla}_{i} \gamma_{r}\right)=\gamma^{r} \bar{\nabla}_{r} \gamma_{i}=c_{i}
$$

$c_{i}$ indicando il veltore di curvatura della linea $x^{0}$,

$$
\mathfrak{B}_{\Sigma \in \mathcal{O}}\left(\Omega_{i j}\right)=c_{i} \gamma_{j}
$$

Viene in tal modo riconosciuto che il vettore spaziale che assieme al tensore spaziale $\tilde{\Omega}_{i j}$, precedentemente considerato, individua il tensore vortice $\boldsymbol{\Omega}_{i j}$ si identifica con il vettore di curvatura della linea $x^{0}$, evidentemente normale alla curva stessa.

Proiezione- $\Theta \Sigma$. - In modo analogo si riconosce, in armonia con quanto si disse al n. 5 per un generico tensore antisimmetrico, che:

$$
\mathfrak{P}_{\circledast \Sigma}\left(\Omega_{i j}\right)=-\gamma_{i} c_{j}
$$

Proiezione- $\Theta \Theta$. - Come per un qualunque tensore antisimmetrico, tale proiezione è identicamente nulla:

$$
\mathfrak{S}_{0 \Theta}\left(\mathbf{Q}_{i j}\right)=0
$$

Quanto precede dà luogo alla seguente decomposizione naturale del ten. sore vortice spaziotemporale $\Omega_{i j}$ :

$$
\Omega_{i j}=\tilde{\Omega}_{i j}+c_{i} \gamma_{j}-\gamma_{i} c_{j}
$$

$\tilde{\Omega}_{i j}$ e $c_{i}$ indicando rispettivamente il tensore vortice spaziale e il vettore di curvatura della congruenza $C_{0}$. 
13. - Le quattro proiezioni naturali del tensore $\bar{\nabla}_{i} \gamma_{j}$. - Cominceremo anzitutto con l'osservare che il secondo indice del tensore $\bar{V}_{i} \gamma_{j}$ e un indice puramente spaziale. Infatti, tenuto presente che $-g_{00}=\gamma_{0}{ }^{2}$, si ha (per ogni valore dell' indice $i$ )

$$
\begin{aligned}
\bar{\nabla}_{i} \gamma_{0} & =\partial_{i} \gamma_{0}-(i o, l) \gamma^{l}=\partial_{i} \gamma_{0}-(i o, o) \gamma^{0} \\
& =\partial_{i} \gamma_{0}-\partial_{i} \gamma_{0}=0 .
\end{aligned}
$$

(Anche più rapidamente si può osservare che, avendo $\gamma$ norma costante, il vettore $\bar{\nabla}_{i} \gamma_{j} d x^{i}$ risulta, qualunque sia $d x^{i}$, ortogonale a $\gamma$ : ne segue che l'indice scoperto $j$ è puramente spaziale). Dall'osservazione ora fatta segue che per ottenere le proiezioni $\Sigma \Sigma$ o $\theta \Xi$ del tensore $\bar{\nabla}_{i} \gamma_{j}$ basta operare sul suo primo indice (rispettivamente col proiettore $\gamma_{i r} 0$ col proiettore $-\gamma_{i} \gamma_{\gamma}$ ) mentre le proiezioni $\Sigma \Theta$ e $\Theta \Theta$ risultano automaticamente nulle.

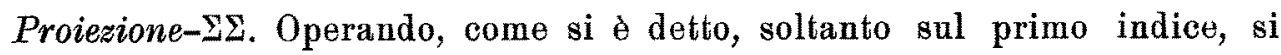
ha successivamente:

$$
\begin{aligned}
\mathfrak{S}_{\Sigma \Sigma}\left(\bar{\nabla}_{i} \gamma_{j}\right) & =\gamma_{i}^{h} \bar{V}_{h} \gamma_{j}=\bar{V}_{i} \gamma_{j}+\gamma_{i} \gamma^{0} \bar{V}_{0} \gamma_{j} \\
& =\partial_{i} \gamma_{j}-(i j, o) \gamma^{0}+\gamma_{i} \gamma^{0} \partial_{0} \gamma_{j}-\gamma_{i} \gamma^{0}(o j, o) \gamma^{0} .
\end{aligned}
$$

Associando il primo e il terzo termine, che insieme danno $\tilde{\partial}_{i} \gamma_{j}$, e sviluppando i simboli di Christoffes, tenendo anche presente che $\gamma_{0} \gamma^{0}=-1, g_{i 0}=-\gamma_{i \gamma_{0}}$, $g_{00}=-\gamma_{0}^{2}$, si trova

$$
\mathfrak{B}_{\Sigma \Sigma}\left(\bar{\nabla}_{i} \gamma_{j}\right)=\tilde{\partial}_{i} \gamma_{j}+\frac{1}{2}\left(\gamma_{0} \partial_{i} \gamma_{j}+\gamma_{j} \partial_{i} \gamma_{0}+\gamma_{0} \partial_{j} \gamma_{i}+\partial_{0} g_{i j}-\gamma_{i} \partial_{j} \gamma_{0}\right) \gamma^{0} .
$$

Converrà far comparire sotto la parentesi i tre termini $\partial_{0}\left(\gamma_{i} \gamma_{j}\right)-\gamma_{i} \partial_{0} \gamma_{j}-\gamma_{j} \partial_{0} \gamma^{i}$ la cui somma è evidentemente nulla. Il primo di essi associato col quarto termine della parentesi da semplicemente $\partial_{0} \gamma_{i j}$. Rimane allora

$$
\mathfrak{B}_{\Sigma \Sigma}\left(\triangle_{i} \gamma_{j}\right)=\tilde{\partial}_{i} \gamma_{j}+\frac{1}{2}\left(\gamma_{0} \partial_{i} \gamma_{j}+\gamma_{j} \partial_{i} \gamma_{0}+\gamma_{0} \partial_{j} \gamma_{i}-\gamma_{i} \partial_{0} \gamma_{j}-\gamma_{j} \partial_{0} \gamma_{i}-\gamma_{i} \partial_{j} \gamma_{0}+\partial_{0} \gamma_{i j}\right) \gamma^{0} .
$$

Entro la parentesi il primo e il quarto termine danno $\gamma_{0} \tilde{\partial}_{i} \gamma_{j}$, il terzo e il quinto danno $\gamma_{0} \tilde{\partial}_{j} \gamma_{i}$, e infine il secondo e il sesto danno $\gamma_{j} \partial_{i} \gamma_{0}-\gamma_{i} \tilde{\partial}_{j} \gamma_{0}$ (infatti $\left.\gamma_{j}\left(\partial_{i} \gamma_{0}+\gamma_{i} \gamma^{0} \partial_{0} \gamma_{0}\right)-\gamma_{i}\left(\partial_{j} \gamma_{0}+\gamma_{j} \gamma^{0} \partial_{0} \gamma_{0}\right) \equiv \gamma_{j} \partial_{i} \gamma_{0}-\gamma_{i} \partial_{j} \gamma_{0}\right)$. Si ha pertanto, sciogliendo la parentesi :

$$
\mathfrak{B}_{\Sigma \Sigma}\left(\triangle_{i} \gamma_{j}\right)=\frac{1}{2} \tilde{\partial}_{i \gamma_{j}}-\frac{1}{2} \tilde{\partial}_{j \gamma_{i}}-\frac{1}{2 \gamma_{0}} \gamma_{j} \tilde{\partial}_{i} \gamma_{0}+\frac{1}{2 \gamma_{0}} \gamma_{i} \tilde{\partial}_{j} \gamma_{0}+\frac{1}{2} \gamma^{0} \partial_{0} \gamma_{i j}
$$


da cui, associando il primo termine col terzo e il secondo col quarto, si ricava infine

$$
\mathfrak{B}_{\mathbb{E}}\left(\triangle_{i} \gamma_{j}\right)=\frac{1}{2} \gamma_{0}\left(\tilde{\partial}_{i} \frac{\gamma_{i}}{\gamma_{0}}-\tilde{\partial}_{j} \frac{\gamma_{i}}{\gamma_{0}}\right)+\frac{1}{2} \gamma^{0} \partial_{0} \gamma_{i j}=\frac{1}{2} \tilde{\boldsymbol{\Omega}}_{i j}+\frac{1}{2} \gamma^{h \partial_{h} \gamma_{i j}}
$$

espressione nella quale figura il tensore antisimmetrico $\tilde{\Omega}_{i j}$ che nel precedente numero abbiamo chiamato il tensore vortice spaziale ed un tensore spaziale simmetrico, $\frac{1}{2} \gamma^{0} \gtrsim_{0} \gamma_{i j} \equiv \frac{1}{2} \gamma^{h} \partial_{h} \gamma_{i j}$, che dovremo esaminare meglio più avanti (n. 14).

A semplice titolo di controllo osserviamo che la (13.3) consente di calcolare in modo immediato la proiezione $\Sigma \Sigma$ del tensore $\Omega_{i j}=3_{i} \gamma_{j}-\partial_{j} \gamma_{i}=\Delta_{i} \gamma_{j}-\Delta_{j} \gamma_{i}$, ottenendo

$$
\mathfrak{S}_{\Sigma \Sigma}\left(\Omega_{i j}\right)=\frac{1}{2}\left(\tilde{\boldsymbol{\Omega}}_{i j}-\tilde{\Omega}_{j i}\right)=\tilde{\Omega}_{i j}
$$

in accordo con la (12.4).

Proiezione-乏 $\Theta$. Tale proiezione, come si è già detto, ¿ nulla, stante il carattere puramente spaziale del secondo indice:

$$
\mathfrak{B}_{\Sigma \in(}\left(\bar{\nabla}_{i} \gamma_{j}\right)=0
$$

Proiezione $-\Theta \Sigma$. Operando solamente sul primo indice, dato il carattere puramente spaziale del secondo, si ottiene

$$
\begin{aligned}
\mathfrak{B}_{\oplus \Sigma}\left(\bar{\nabla}_{i} \gamma_{j}\right) & =-\gamma_{i} \gamma^{r} \bar{\nabla}_{r} \gamma_{j}=-\gamma_{i} \gamma^{0}\left[\partial_{0} \gamma_{j}-(o j, h) \gamma^{h}\right] \\
& =-\gamma_{i} \gamma^{0}\left[\partial_{0} \gamma_{j}-\partial_{j} \gamma_{0}\right] \\
& =-\gamma_{i} \gamma^{r} \Omega_{r j} .
\end{aligned}
$$

In definitiva risulta:

$$
\begin{aligned}
\mathfrak{B}_{\Theta \Sigma}\left(\bar{\nabla}_{i} \gamma_{j}\right) & =-\gamma_{i} \gamma^{r} \Omega_{r j} \\
& =-\gamma_{i} c_{j}
\end{aligned}
$$

che coincide con la proiezione- $\Theta \boldsymbol{Q}$ del tensore $\boldsymbol{\Omega}_{i j}$.

Proiezione- $\theta \Theta$. Anche questa proiezione si ̀̀ già detto essere nulla, a causa del carattere puramente spaziale del secondo indice di $\Lambda_{i} \gamma_{j}$.

La decomposizione naturale del tensore $\Lambda_{i} \gamma_{j}$ pnò allora cosi riassumersi

$$
\begin{aligned}
\Delta_{i \gamma_{j}} & =\frac{1}{2} \tilde{\Omega}_{i j}+\frac{1}{2} \gamma^{h} \partial_{h \gamma_{i j}}-\gamma_{i} \gamma^{r} \Omega_{r j} \\
& =\frac{1}{2}\left(\tilde{\Omega}_{i j}+\gamma^{h} \partial_{h} \gamma_{i j}\right)-\gamma_{i} c_{j} .
\end{aligned}
$$


14. - Proiezioni naturali del tensore di Killing: $K_{i j} \equiv \bar{\nabla}_{i} \gamma_{j}+\bar{\nabla}_{j} \gamma_{i} .-$ Un altro tensore ben noto, relativo ad una generica congruenza $C_{0}$, $\theta$ della più grande importanza, è il tensore doppio simmetrico $K_{i j}=\bar{\nabla}_{i} \gamma_{j}+\bar{\nabla}_{j} \gamma_{i}$, al quale sembra ben appropriato il nome di tensore di Killing (cfr. [10] [6]). Come è ben noto, l' annullarsi identico del tensore $K_{i j}$ esprime la condizione necessaria e sufficiente perehè la congruenza $C_{0}$ definisca in $V_{n+1}$ un gruppo di isometrie (a un parametro).

Le proiezioni naturali di detto tensore si ottengono immediatamente dalle analoghe proiezioni del tensore $\bar{\nabla}_{i} \gamma_{j}$ (cfr. n. precedente). Ecco quel che si ottiene :

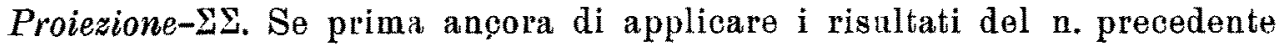
si applica ai due indioi di $K_{i j}$ la regola formale di proiezione su $\Sigma$, si ottiene

$$
\begin{gathered}
\mathfrak{S}_{\mathbf{I} \Sigma}\left(\bar{\nabla}_{i} \gamma_{j}+\bar{\nabla}_{j} \gamma_{i}\right) \equiv \tilde{K}_{i j}=\left(\partial_{i}{ }^{h}+\gamma_{i} \gamma^{h}\right) \bar{\nabla}_{h} \gamma_{j}+\left(\partial_{j}{ }^{k}+\gamma_{j} \gamma^{k}\right) \nabla_{k} \gamma_{i}= \\
=\bar{\nabla}_{i} \gamma_{j}+\bar{\nabla}_{j} \gamma_{i}+\gamma_{i} \gamma^{h} \bar{\nabla}^{h} \gamma_{j}+\gamma_{j} \gamma^{h} \bar{\nabla}^{h} \gamma_{i}
\end{gathered}
$$

ove all' ultimo membro si riconosce il tensore spaziale simmetrico introdotio in relatività da Bonn fin dal 1909 (cfr. Born [1], Syna [17] SalzmanN e TAUB [16] e molti altri autori). Conformemente alle nostre convenzioni generali noi indicheremo tal tensore anche con la notazione $\tilde{K}_{i j}$, attribuendogli nel contempo il nome di tensore spaziale di Born. Il suo annullarsi identico, meno restrittivo dell'annullarsi identico dell'intero tensore di KILLING $K_{i j}$, esprime che il moto fluido individuato dalla congruenza $C_{0}$ delle linee $x^{0} \dot{e}$ rigido secondo BonN ([1]) cioè la costanza della distanza spaziale tra due qualunque linee $x^{0}$ infinitamente vicine (cfr. Srnge [17] p. 36). Nel caso che esso non sia identicamente nullo, esso può ben interpretarsi come tensore di deformazione del fluido $C_{0}$, ed è proprio su tale tensore che si fondano $i$ vari tentativi (Herglotz [9], Synge [18], Benvenomi (i)) di una teoria relativistica della elasticità.

Venendo ora ad una più precisa esplicitazione della $\mathfrak{B}_{z \Sigma}\left(K_{i j}\right)$ si riconosce immediatamente, in base alla (13.3), e all'antisimmetria del tensore vortice spaziale $\mathbf{\Omega}_{i j}$, l'espressione semplicissima

$$
\mathfrak{S}_{\Sigma \Sigma}\left(K_{i j}\right) \equiv \tilde{K}_{i j}=\gamma^{h} \partial_{h} \gamma_{i j}
$$

Su tale espressione si può ben osservare come l'annullarsi identico del tensore di BorN, equivalga in sostanza all'indipendenza da $x^{0}$ del tensore

(1) P. Benvenuti, tesi di laurea (1956). 
metrico spaziale $\gamma_{i j}$ (che non è la stessa cosa della stazionarietà) indipendenza da $x^{0}$ del tensore metrico spazio temporale $g_{i j}$.

Quanto alle altre proiezioni naturali si ha:

\section{Proiezione-20:}

$$
\mathscr{B}_{\mathbb{\Theta \Theta}}\left(K_{i j}\right)=-c_{i} \gamma_{j}
$$

Proiezione- $\Theta \mathbf{\Sigma}$ :

$$
\mathfrak{S}_{\mathrm{\Sigma} \Theta}\left(K_{i j}\right)=-\gamma_{i} c_{j}
$$

Proiezione- $\Theta \Theta$ :

$$
\mathfrak{S}_{\oplus \mathscr{G}}\left(K_{i j}\right)=0
$$

Come nei precedenti numeri, $c_{i}$ rappresenta il vettore di ourvatura (ortogonale a $\gamma$ ) delle linee $x^{0}$.

La decomposizione naturale del tensore di KILLING si esplicita in definitiva così :

$$
K_{i j}=\tilde{K}_{i j .}-c_{i} \gamma_{j}-\gamma_{i} c_{j}
$$

15. Alcune proprietà differenziali della congruenza $C_{0}$. Nei tensori presi in considerazione nei precedenti numeri, o in alcune loro proiezioni naturali sono contenute notevoli proprietà differenziali della congruenza $C_{0}$, inerenti ciò̀ al modo di variare di $\gamma$ da un punto all'altro di $V_{n+1}$. Si tratta di proprietà note, ma ci sembra che la formulazione attuale dia loro una sistemazione più organica e stabilisca tra esse un naturale collegamento.

Congruenze normali. Condizione necessaria e sufficiente perchè $C_{0}$ sia una congruenza normale è l'annullarsi idèntico del suo vortice spaziale

$$
\mathfrak{S}_{\Sigma \Sigma}\left(\mathbf{\Omega}_{i j}\right) \equiv \tilde{\Omega}_{i j} \equiv \gamma_{0}\left(\tilde{\partial}_{i} \frac{\gamma_{j}}{\gamma_{0}}-\tilde{\mathfrak{\partial}}_{j} \frac{\gamma_{i}}{\gamma_{0}}\right)=0
$$

Il teorema, in forma sostanzialmente equivalente, è dovato a WEISSENHoFF (cfr. [19] e [14] p. 250). Per riconoscerne la validita ricordiamo che tradizionalmente (cfr. Levi-Civita, [11] p. 281) la normalità di una congruenza si esprime mediante l'annullarsi identico del tensore triplo

$$
\gamma_{i} \boldsymbol{Q}_{j k}+\gamma_{j} \mathbf{Q}_{k i}+\gamma_{k} \mathbf{Q}_{i j}=0
$$

col solito significato di $\gamma_{i}$ e $\Omega_{i j}$. Ora è semplice riconoscere la completa equivalenza delle condizioni (15.1) e (15.2). Infatti se nei vari termini della 
(15.2) il tensore $\Omega_{i j}$ viene decomposto nei suoi componenti naturali (cfr. la (12.8)) si ottiene successivamente

$$
\gamma_{i}\left(\tilde{\Omega}_{j k}+c_{j} \gamma_{k}-\gamma_{j} c_{k}\right)+\gamma_{j}\left(\tilde{\Omega}_{k i}+c_{k} \gamma_{i}-\gamma_{k} c_{i}\right)+\gamma_{k}\left(\tilde{\Omega}_{i j}+c_{i} \gamma_{j}-\gamma_{i} c_{j}\right)=0
$$

cosicehè la (15.2) appare completamente equivalente all'altra condizione

$$
\gamma_{i} \tilde{\boldsymbol{\Omega}}_{j k}+\gamma_{j} \tilde{\boldsymbol{\Omega}}_{k i}+\gamma_{k} \tilde{\mathbf{Q}}_{i j}=0 .
$$

Di qui, moltiplicando i due membri per $\gamma^{i}$ (o per $\gamma^{i}$ o per $\gamma^{k}$ ) e saturando l'indice muto $i$ (o rispettivamente $j \circ k$ ) discende $\tilde{\Omega}_{j k}=0$, ciò̀ esattamente la (15.1). Viceversa dalla (15.1) discende immediatamente la (15.3) e quindi la condizione classica (15.2).

Congruenze geodeliche. Condizione necessaria e sufficiente affinchè la congruenza $C_{0}$ sia una congrnenza geodetica, affinch ò cioè siano geodetiche le linee che la costituiscono, è che sia nulla la proiezione- $₫ \Theta$ (e quindi anche la proiezione- $\Theta \Xi$ ) del suo tensore vortice $\Omega_{i j}$ :

$$
\mathfrak{B}_{\mathbf{\Sigma}}\left(\mathbf{Q}_{i j}\right)=0 .
$$

Infatti dalla (12.5) segue che $\mathfrak{B}_{\Sigma \Theta}\left(\boldsymbol{\Omega}_{i j}\right)$ si annulla allora e allora soltanto che si annulla $c_{i}$, vettore di curvatura delle linee $x^{0}$.

Congruenze geodetiche e normali. Associando le due osservazioni precedenti si ricava che condizione necessaria e sufficiente perchè la congruenza $C_{0}$ sia insieme normale e geodetica è che sia nullo identicamente il suo vettore vortice:

$$
\Omega_{i j}=0 \text {. }
$$

D'altro canto la condizione ora scritta dà anche quanto cccorre e basta perchè il campo di versori $\gamma$ sia un campo di gradienti: $\gamma=$ grad $f$. Si tratta evidentemente di un campo di gradienti assai particolare, nel quale due qualunque superficie equipotenziali sono superficie parallele (cioè tali che le geodetiche normali ad una di esse sono normali anche all'altra, i segmenti intercettati dalle due superficie sulle varie geodetiche avendo tutti una medesima lunghezza).

Congruenze definenti movimenti rigidi. Segne da quanto si disse al $\mathrm{n} .14$ che condizione necessaria e sufficiente perchè il movimento continuo definito dalla congruenza $C_{0}$ sia rigido secondo BonN, perchè cioè la distanza tra due linee $x^{0}$ qualsiasi infinitamente vicive, misurata su una comune normale, rimanga costante lungo le linee medesime ò che sia ovunque nullo il tensore di BORN:

$$
\mathfrak{S}_{\Sigma \Sigma}\left(K_{i j}\right) \equiv \tilde{K_{i j}} \equiv \gamma^{0} \partial_{0} \gamma_{i j}=0
$$




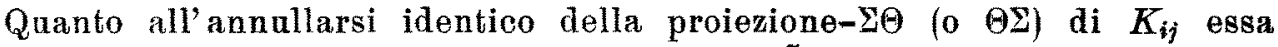
caratterizza, proprio come l'annullarsi identico di $\tilde{\mathbf{\Omega}}_{i}$, le congruenze geodetiche.

Congruenze rigide e geodetiche insieme. Sono caratterizzate, in base a quanto precede, dall' annullarsi identico dell' intero tensore di KILLING:

$$
K_{i j} \equiv \nabla_{i} \gamma_{j}+\nabla_{j} \gamma_{i}=0 \text {. }
$$

D'altro canto, come è ben noto, la condizione (15.7) caratterizza quelle congruenze che definiscono un gruppo di isometrie in $V_{n+1}$ (ofr. EIsenharT [6], LtonNerowioz [13]). Noi diremo che l'annullarsi identico del tensore di KruLING caratterizza i moti rigidi e geodetici.

Moti rigidi traslatori uniformi. Associando insieme le considerazioni che precedono, si riconosce che l'annullarsi identico del tensore $\nabla_{i} \gamma_{j}$, che implica insieme l'annullamento di $K_{i j}$ e di $\Omega_{i j}$ earatteriza quei moti che sono ad un tempo irrotazionali, rigidi e geodetici:

$$
\nabla_{i} \gamma_{j}=0
$$

Li chiameremo moti traslatori uniformi.

Quanto precede mi sembra mostrare a sufficienza come il criterio della decomposizione naturale dei principali tensori differenziali associati ad una congruenza del genere tempo consenta una sistematica classificazione dei possibili moti fluidi. Benchè non si tratti di risultati nuovi, mi sembra non priva di interesse questa loro più organica sistemazione.

IV. - DeRIVAzIone trastersa, ordinatia o covariante, di partiCOLARI CAMFI TENSORIALI ED INTERVENTO DEI TENSORI DIFFERENZIALI CARATTERISTICI DELLA CONGRUENZA DI RIFERIMENTO.

Riprendiamo qui il calcolo esplicito, iniziato al precedente n. 8, della derivata covariante trasversa di vari campi di tensori puramente spaziali, le cui espressioni esplicite acquistano. snellezza ed espressività mediante l'intervento dei tensori differenziali caratteristici della congruenza principale di riferimento. Tale intervento esplicito consente infatti di distinguere nelle espressioni medesime ciò che è dovuto alla congruenza di riferimento e ciò che invece è dovuto al campo tensoriale che si deriva.

Come vedremo, anche le regole puramente formali relative alla derivazione trasversa sono talora subordinate alla scelta della congruenza principale di riferimento. Ne daremo subito un esempio.

16. Condizioni per l'invertibilità di due derivazioni trasverse (ordinarie) successive. Vedemmo fin dal $\mathrm{n}$. 7 che due derivazioni trasverse successive non sono generalmente invertibili. Stabiliremo ora sotto quali condizioni l' invertibilità sussiste.

Data una qualsiasi funzione numerica $f\left(x^{i}\right)$ (sia essa o no uno scalare) 
consideriamone, formalmente, la derivata trasversa seconda $\tilde{\tilde{c}}_{p} \tilde{\partial}_{\alpha} f$ che si espli. cita cosl:

$$
\begin{aligned}
\tilde{\partial}_{\beta} \tilde{\partial}_{\alpha} f & =\left(\partial_{\beta}-\frac{\gamma_{\beta}}{\gamma_{0}} \partial_{0}\right)\left(\partial_{\alpha} f-\frac{\gamma_{\alpha}}{\gamma_{0}} \partial_{0} f\right)= \\
& =\partial_{\beta} \partial_{\alpha} f-\frac{\gamma_{\beta}}{\gamma_{0}} \partial_{0} \partial_{\alpha} f-\left(\partial_{\beta} \frac{\gamma_{x}}{\gamma_{0}}-\frac{\gamma_{\beta}}{\gamma_{0}} \partial_{0} \frac{\gamma_{\alpha}}{\gamma_{0}}\right) \partial_{0} f- \\
& -\frac{\gamma_{\alpha}}{\gamma_{0}} \partial_{\beta} \partial_{0} f+\frac{\gamma_{\alpha} \gamma_{\beta}}{\gamma_{0}{ }^{2}} \partial_{0}^{q} f .
\end{aligned}
$$

In modo analogo, invertendo l'ordine delle due derivazioni si ottiene:

$$
\begin{aligned}
\tilde{\partial}_{\alpha} \tilde{\partial}_{\beta} f & =\partial_{\alpha} \partial_{\beta} f-\frac{\gamma_{\alpha}}{\gamma_{0}} \partial_{0} \partial_{\beta} f-\left(\partial_{\alpha} \frac{\gamma_{\beta}}{\gamma_{0}}-\frac{\gamma_{\alpha}}{\gamma_{0}} \partial_{0} \frac{\gamma_{\beta}}{\gamma_{0}}\right) \partial_{0} f- \\
& -\frac{\gamma_{\beta}}{\gamma_{0}} \partial_{\alpha} \partial_{0} f+\frac{\gamma_{\beta} \gamma_{\alpha}}{\gamma_{0}{ }^{2}} \partial_{0}^{2} f .
\end{aligned}
$$

Dalle due espressioni per differenza si ottiene

$$
\left(\tilde{\partial}_{\beta} \tilde{\partial}_{\alpha}-\tilde{\partial}_{\alpha} \tilde{\partial}_{\beta}\right) f=\left(\tilde{\partial}_{\alpha} \frac{\gamma_{\beta}}{\gamma_{0}}-\tilde{\partial}_{\beta} \frac{\gamma_{\alpha}}{\gamma_{0}}\right) \partial_{0} f \text {. }
$$

Se ne conclude che l'ordine di due derivazioni trasverse successive non influisce sul risultato se il moto di riferimento è irrotazionale (spazialmente) oppure se $f(x)$ non dipende $d a x^{0}$ ( $f$ stazionaria lungo ogni linea di riferimento $x^{0}=$ var.).

17. Condiaione per l'invertibilità $d i \tilde{\partial}_{\alpha} e \partial_{0}$. Applicando successivamente ad una funzione $f\left(x^{i}\right)$ i due operatori $\partial_{0}$ e $\tilde{\partial}_{x}$ si ha:

$$
\tilde{\partial}_{\alpha} \partial_{0} f=\partial_{\alpha} \partial_{0} f-\frac{\gamma_{\alpha}}{\gamma_{0}} \partial_{0}^{2} f .
$$

Applicando i due operatori in ordine inverso si ha invece:

$$
\partial_{0} \tilde{\partial}_{\alpha} f=\partial_{0} \partial_{\alpha} f-\partial_{0}\left(\frac{\gamma_{\alpha}}{\gamma_{0}}\right) \partial_{0} f-\frac{\gamma_{\alpha}}{\gamma_{0}} \partial_{\alpha}^{2} f
$$

Per differenza si ottiene:

$$
\tilde{\partial}_{\alpha} \partial_{0} f-\partial_{0} \tilde{\partial}_{\alpha} f=\partial_{0}\left(\frac{\gamma x}{\gamma_{0}}\right) \cdot \partial_{0} f
$$

Ne concludiamo che gli operatori differenziali $\partial_{0}$ e $\tilde{\partial}_{\alpha}$ sono commutabili soltanto se la $f$ è stazionaria in $C_{0}$ (cioè se non dipende da $x^{0}$ ), oppure se è stazionario il «potenziale gravitazionale vettore $\frac{\gamma_{\alpha}}{\gamma_{0}}$.

18. Proiezioni naturali $d i \bar{\nabla}_{i} \tau_{j}\left(\tau_{j} \in \Theta_{x}\right)$. Sia $\tau_{j}=\tau \gamma_{j}$ un generico campo di 
vettori puramente temporali. Considerata la sua derivata covariante $\bar{\nabla}_{i} \tau_{j}$, calcoliamone le sue quattro proiezioni naturali.

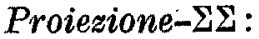

$$
\begin{aligned}
\mathfrak{B}_{\Sigma \Sigma}\left(\bar{\nabla}_{i} \tau_{j}\right) & =\gamma_{i}{ }^{r} \gamma_{j}{ }^{s} \partial_{r} \tau \cdot \gamma_{s}+\tau \mathfrak{B}_{\Sigma \Sigma}\left(\nabla_{i} \gamma_{j}\right)= \\
& =\tau \mathfrak{S}_{\Sigma \Sigma}\left(\bar{\nabla}_{i} \gamma_{j}\right) .
\end{aligned}
$$

Di qui, tenendo conto della (13.3) si trae:

$$
\begin{aligned}
\mathfrak{S}_{\Sigma \Sigma}\left(\bar{\nabla} i \tau_{j}\right) & =\frac{1}{2} \tau\left(\gamma^{h} \partial_{0} \gamma_{i j}+\tilde{\Omega}_{i j}\right)= \\
& =\frac{1}{2} \tau\left(\tilde{K}_{i j}+\tilde{\Omega}_{i j}\right) .
\end{aligned}
$$

Si osservi come la proiezione ora calcolata dipenda oltre che dal campo di vettori $\tau_{j}$, dalla congruenza di riferimento $C_{0}$. Se questa, ad esempio, è ad un tempo rigida e irrotazionale, la proiezione (18.2) si annulla identicamente, qualunque sia il campo dei vettori puramente temporali $\tau_{j}$.

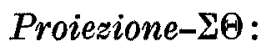

$$
\begin{aligned}
& \mathfrak{S}_{\Sigma(\Theta)}\left(\nabla \tau_{j}\right)=-\gamma_{i}{ }^{r} \gamma_{j} \gamma^{8} \partial_{r} \tau \cdot \gamma_{s}+\tau \mathfrak{B}_{\Sigma \Theta}\left(\nabla_{i} \gamma_{j}\right)= \\
& =\tilde{\partial}_{i} \tau \cdot \gamma_{j}+\tau \mathfrak{B}_{\mathrm{E}}\left(\bar{\nabla}_{i} \gamma_{j}\right) .
\end{aligned}
$$

Se si tien presente la (13.4) si conclude:

$$
\mathscr{S}_{\Sigma \omega}\left(\nabla_{i} \tau_{j}\right)=\tilde{\partial}_{i} \tau \cdot \gamma_{j}
$$

Proiezione-0ะ:

$$
\begin{aligned}
\mathfrak{B}_{\Theta \mathrm{X}}\left(\bar{\nabla}_{i} \tau_{j}\right) & =\gamma_{i} \gamma^{r} \gamma_{j}{ }^{s} \partial_{r} \tau \cdot \gamma_{s}+\tau \mathfrak{B}_{\Theta \mathrm{E}}\left(\nabla_{i} \gamma_{j}\right)= \\
& =\tau \mathfrak{S}_{\Theta \Sigma}\left(\nabla_{i} \gamma_{j}\right)
\end{aligned}
$$

e, ricordando la (13.5),

$$
\mathfrak{B}_{\circledast \Sigma}\left(\nabla_{i} \tau_{j}\right)=-\tau \gamma_{i} c_{j} .
$$

Tal proiezione si annulla identicamente, qualunque sia il campo dei vettori puramente temporali $\tau_{j}$, se $C_{0}$ \& una congruenza geodetica.

Proiezione- $\Theta \Theta$ :

$$
\mathscr{B}_{\oplus \Theta}\left(\bar{\nabla}_{i} \tau_{j}\right)=\gamma_{i} \gamma_{j} \gamma^{r_{\gamma}}{ }^{s} \partial_{r} \tau \cdot \gamma_{s}+\tau \mathfrak{B}_{\Theta \Theta}\left(\nabla_{i} \gamma_{j}\right) .
$$

Cio permette di concludere:

$$
\mathfrak{B}_{\Theta \Theta}\left(\nabla_{i} \tau_{j}\right)=-\gamma^{0} \partial_{0} \tau \cdot \gamma_{i} \gamma_{j}=-\gamma^{r} \partial_{r} \tau \cdot \gamma_{i} \gamma_{j} .
$$

La $\mathfrak{B}_{\Theta \Theta}\left(\bar{V}_{i} \tau_{j}\right)$ si annulla allorchè il campo $\tau_{j}$ è stazionario, ciò̀ allorchè $\tau_{j} \grave{\partial}$ costante in modulo lungo ogni linea di $C_{0}$. 
19. Proiezioni naturali $d i \bar{\nabla}_{i} s_{i}\left(s_{j} \in \Sigma_{x}\right)$. Calcoliamo ora le quattro proiezioni naturali del tensore $\bar{V}_{i} s_{j}$ nell'ipotesi che $s_{j}$ sia un campo di vettori puramente spaziali. L'ipotesi equivale a supporre, come ben sappiamo,

$$
s_{0}=0, \quad s^{0}=-\frac{\gamma_{\alpha} s^{x}}{\gamma_{0}} \equiv \gamma^{0} \gamma_{\mathcal{L}} s^{x} .
$$

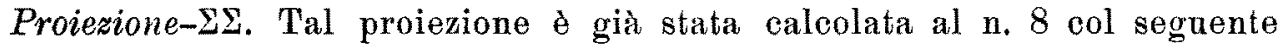
risultato (cfr. la (8.4)):

$$
\mathfrak{B}_{\Sigma \Sigma}\left(\bar{\nabla}_{i} s_{j}\right)=\tilde{\bar{V}}_{i}^{*} s_{j}=\tilde{\partial}_{i} s_{j}-\left\{\begin{array}{l}
\tilde{h} \\
i j
\end{array}\right\} s_{h}
$$

$\tilde{\bar{V}}_{i}^{*}$ indicando l'operazione di derivazione covariante trasversa. Occupiamoci ora delle altre proiezioni.

Proiezione- $\Sigma \Theta$ :

Delle altre proiezioni naturali di $\bar{\nabla} s_{j}$ ei limitiamo a dare $\mathrm{i}$ risultati, omettendo i calcoli, piuttosto lunghi. Ecco le espressioni finali

$$
\begin{aligned}
& \mathscr{S}_{\Sigma \otimes}\left(\bar{\nabla}_{i} s_{j}\right)=-\frac{1}{2}\left(\tilde{K}_{i r}+\tilde{\Omega}_{i r}\right) s^{r_{\gamma_{i}}} . \\
& \mathfrak{B}_{\Theta \Sigma}\left(\nabla_{i} s_{j}\right)=\gamma_{i}\left[\gamma^{h}\left(\partial_{j} s_{h}-\partial_{h} s_{j}\right)+\frac{1}{2}\left(\tilde{K}_{j r}+\tilde{Q}_{j r}\right) s^{r}\right] . \\
& \mathfrak{S}_{\Theta \Theta}\left(\bar{V}_{i} s_{j}\right)=\left(\Omega_{r} s^{r} \gamma^{h}\right) \gamma_{i} \gamma_{j}=-c_{r} s^{r} Y_{i} \gamma_{j} .
\end{aligned}
$$

20. Proiezioni naturali $d i \bar{\nabla}_{i} s_{j m}\left(s_{j m} \in \Sigma_{x} \otimes \Sigma_{x}\right)$. Diamo qui appresso la decomposizione naturale del tensore triplo $\bar{V}_{i} s_{j m}, s_{j m}$ essendo un generico campo di. vettori puramente spaziali $\left(s_{j 0}=0, s_{0 m}=0\right)$. Omettiamo anche ora. per brevità, i lunghi calcoli.

Ecco le otto proiezioni naturali di $\bar{V}_{i} s_{j m}$ :

$$
\begin{aligned}
& \mathfrak{S}_{\Sigma \Sigma \Sigma}\left(\bar{\nabla}_{i} s_{j m}\right)=\tilde{\bar{V}}_{i}^{*} s_{j m} \equiv \tilde{a}_{i} s_{j m}-\left\{\begin{array}{c}
h \\
i j
\end{array}\right\} s_{h m}-\left\{\begin{array}{c}
h \\
i m
\end{array}\right\} s_{j h} . \\
& \mathfrak{B}_{\Sigma \Sigma \mathcal{O}}\left(\bar{\nabla}_{i} s_{j m}\right)=\frac{1}{2} \tilde{\mathbb{Q}}_{i k} s_{j}^{k} \gamma_{m}+\frac{1}{2} \gamma^{0} \partial_{0} \gamma_{i k} s_{j}{ }^{k} \gamma_{m} . \\
& \mathfrak{B}_{\Sigma(\Theta \Sigma}\left(\bar{\nabla}_{i} s_{j m}\right)=\frac{1}{2} \tilde{\Omega}_{i k} \gamma_{j} s^{k}{ }_{m}+\frac{1}{2} \gamma^{0} \mathfrak{c}_{0} \gamma_{i k} s^{k}{ }_{m} \gamma_{j} .
\end{aligned}
$$

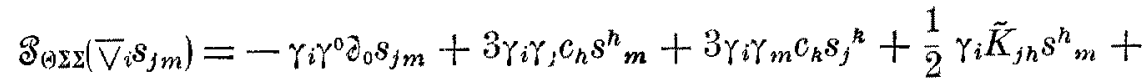

$$
\begin{aligned}
& +\frac{1}{2} \gamma_{i} \tilde{K}_{m k} s_{j}{ }^{k}+\gamma_{i} \tilde{\mathbf{Q}}_{h j} s^{h}{ }_{m}+\gamma^{i} \tilde{\mathbf{S}}_{h m} s_{\xi}{ }^{k} \text {. }
\end{aligned}
$$




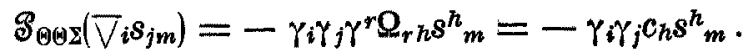

$$
\begin{aligned}
& \mathfrak{B}_{\Theta \varepsilon \Theta}\left(\nabla_{i} s_{j m}\right)=-\gamma_{i} c_{h} s_{j}{ }^{h} \gamma_{m} . \\
& \mathfrak{S}_{\Sigma \Theta \Theta}\left(\bar{V}_{i} s_{j m}\right)=0 . \\
& \mathfrak{S}_{\Theta \oplus \Theta}\left(\bar{\nabla}_{i} s_{j m}\right)=0 .
\end{aligned}
$$

21. - Considerazioni conclusive. - I calcoli sistematici che precedono ci mettono in condizione di svolgere in modo pressochè automatico le seguenti operazioni :

a) decomporre in modo.canonico (naturale) un qualsiasi vettore o tensore.

b) calcolare le proiezioni naturali della derivata covariante di un qualsiasi campo di vettori o tensori doppi, previa applicazione della decomposizione medesima al vettore o tensore di partenza. Infatti questa operazione preventiva permette di ricondursi sempre al caso in cui la derivazione cova. riante operi su vettori puramente spaziali o puramente temporali, ovvero su tensori doppi puramente spaziali.

Il lettore non avrà poi difficoltà ad estendere quanto precede al caso che l'operazione di derivazione covariante operi su tensori di ordine superiore al secondo.

Ritengo con quanto precede di avere a sufficienza illustrato, anche nella sua parte esecutiva, una tecnica che, per il suo carattere invariantivo di fronte al più generale cambiamento interno di coordinate, e per la sua semplicità formale, può apportare utili servigi sia in geometria riemanniana sia, più specificamente, in relatività generale.

\section{BIBLIOGRAFIA}

[1] Born, M., * Ann. Physík * (4), 30, 1 (1909).

[2] Campaneo, O., Nuovo Cimento :, 10, 318 (1958).

[3] - - Nuovo Cimento », 11, 733 (1959).

[4] - Comptes Rendus s, 248, 197 (1959).

[5] - - Nuovo Cimento *, 12 (giugno 1959).

[6] Ersenhart, L. P., Riemannian Geometry, Princeton University Press *, Princeton (1949).

[7] Finzi, B. e M. PAstori, Caleolo tensoriale e applicazioni, Zanichelli, Bologna (1949).

[8] Göper, K., Proe. of the Sixth Intern. Congr. of Math. (Amer. Math. Soc., Providenec, $1950)$, vol. 1, p. 175. 
[9] Heralotz, G., Ann. Physik > (4), 31, 393 (1909-1910).

[10] Killing, W., Journal für die reine und angew. Math. (Crelle), 109, 121 (1892).

[11] Lovi-Civita, T., Lezioni di calcolo differenziale assoluto, Stock, Roma (1925).

[12] Lichnerowioz, A., Éléments de Caleul tensoriel, Colin, Paris (1955).

[13] - - Théories relativistes de la gravitation et de l'electromagnétisne, Masson, Paris, (1955).

[14] Molcen, C., The theory of Relativity, Clarendon Press, Oxford (1952).

[15] Noether, F., Ann. Physik *, 81, 919 (1909-1910).

[16] Salzman, G. e A. H. Taub, *Phys. Rev. *, 95, 1659 (1954).

[17] Synge, J. L., Relativity: The Special Theory, North-Holland Publishing Company, Amsterdam (1956).

[18] - - The Geometry of Space-Time (Eight Lectures on Relativity) in * Vedute e problemi attuali in relativita generale *, C.I.M.E., Istituto Matematico dell'Università di Roma (1958).

[19] V. Weyssenhoff, J., Bull. Acad. Polonaise *, Ser. A, 252 (1987). 